\title{
A case of small-cell lung cancer with adrenocorticotropic hormone deficiency induced by nivolumab
}

This article was published in the following Dove Medical Press journal:

OncoTargets and Therapy

\author{
Yan Zhu' \\ Hong Hua $\mathrm{Wu}^{2}$ \\ Wei Wang ${ }^{2}$ \\ 'Department of Oncology, Peking \\ University First Hospital, Beijing, \\ China; ${ }^{2}$ Department of Endocrinology, \\ Peking University First Hospital, \\ Beijing, China
}

\begin{abstract}
Anti-programmed death-1 (anti-PD-1) monoclonal antibodies, such as nivolumab, have been used for the treatment of various types of cancers, and excellent efficacy has been shown in some patients. The adverse effects of anti-PD-1 antibodies relating to autoimmunity are different from traditional chemotherapeutic drugs and may involve many organs including the endocrine system. We herein describe a case of adrenocorticotropic hormone deficiency during the treatment of advanced small-cell lung cancer, probably caused by nivolumab-induced hypophysitis. The case showed nonspecific, insidious, as well as potentially life-threatening characters of immune-related adverse effects. It is important for physicians to acknowledge clinical features of the rare side effect and take appropriate and prompt treatment.
\end{abstract}

Keywords: PD-1 inhibitor, ACTH deficiency, immune checkpoint inhibitor, hypophysitis, immune-related adverse events

\section{Introduction}

Traditional chemotherapeutic drugs have been dominant agents in cancer therapy for a long time. But chemotherapy resistance occurs inevitably in most advanced malignancies. Programmed death-1 (PD-1) and cytotoxic T-lymphocyte antigen-4 (CTLA-4) are inhibitory immune receptors that are expressed on the surface of cytotoxic $\mathrm{T}$ cells, interacting with their ligands: programmed death-ligand 1 and CD80/CD86, respectively. The binding of the ligand to the receptor inhibits immune response of $\mathrm{T}$ cells to cancer cells. Anti-PD-1 antibodies such as nivolumab and anti-CTLA-4 antibody such as ipilimumab are immune-activating agents named immune checkpoint inhibitors (ICPis), which antagonize the binding between the ligands and the receptors, resulting in T-cell reactivation and attack of cancer cells. ${ }^{1}$ Anti-PD-1 antibodies have been used to treat various neoplasms in recent years, and the effects are excellent in some cases. ${ }^{2}$

Although clinically effective, anti-PD-1 antibodies may cause organ- or tissuespecific immune reactions involving gastrointestinal tract, liver, lung, skin, endocrine system, etc. ${ }^{3-5}$ As the adverse effects may be life-threatening, accurate diagnosis and appropriate treatment are very crucial. Adrenocortical insufficiency is one of the adverse effects involving the endocrine system. ${ }^{3-5}$ The symptoms are nonspecific and insidious, and delaying diagnosis may lead to serious situations in patients.

Here, we describe a patient with advanced small-cell lung cancer (SCLC) who presented with symptoms similar to the general manifestations of malignancies during treatment with nivolumab. Laboratory tests indicated the diagnosis of adrenocorticotropic hormone (ACTH) deficiency, probably caused by nivolumab-induced
Department of Oncology, Peking University First Hospital, Xishiku Street No. 8, Xicheng District, Beijing 100034, China

Tel +8601064002079

Fax +8601084018352

Email zhuyan0302@I26.com 
hypophysitis. We describe the case in detail and summarize the characteristics of ACTH deficiency caused by ICPis including nivolumab.

\section{Case report}

A 61-year-old Chinese male was admitted to our hospital with complaints of hemoptysis and shortness of breath. Chest computed tomography (CT) showed a mass in the upper lobe of right lung. SCLC was diagnosed by bronchoscopic biopsy. In the first 9-month duration after diagnosis, he received multiple lines of chemotherapy sequentially including the combination of etoposide and cisplatin, paclitaxel and irinotecan treatment, as well as radiotherapy for the thoracic tumor. Then, he was followed up regularly. Three months after finishing the last round of chemotherapy, he felt short of breath again. Chest CT showed aggravated atelectasis. Physical examination and CT revealed an enlarged right supraclavicular lymph node. The patient then received treatment of nivolumab with $3 \mathrm{mg} / \mathrm{kg}$ every 2 weeks in 12 doses, for a total of 9 months. Two weeks after the start of nivolumab therapy, his shortness of breath was alleviated. Four weeks later, physical examination and CT showed that the enlarged lymph node shrank significantly (Figure 1), and CT showed that the atelectasis of the right lung was stable.

However, the patient complained of anorexia, fatigue, and vomiting 13 weeks after the initial nivolumab therapy and was admitted to the emergency department. Physical examination showed his body temperature was $38^{\circ} \mathrm{C}$ and blood pressure was 91/61 mmHg. Electrocardiogram showed sinus tachycardia. Laboratory tests showed hyponatremia with serum sodium of $115 \mathrm{mmol} / \mathrm{L}$, and blood glucose was normal. C-reactive protein (CRP) was $200 \mathrm{mg} / \mathrm{L}$ (normal range: $<3 \mathrm{mg} / \mathrm{L}$ ), and the ratio of eosinophils was $8.5 \%$ ( $1.6 \%$ before nivolumab treatment; normal range: $0.4 \%-8 \%$ ), while leukocyte and neutrophil counts were normal. Serum triiodothyronine (T3) (normal range: $0.92-2.79 \mathrm{nmol} / \mathrm{L}$ ) was elevated slightly at $2.94 \mathrm{nmol} / \mathrm{L}$, tetraiodothyronine (T4), free triiodothyronine (FT3), free tetraiodothyronine (FT4), and thyroid-stimulating hormone (TSH) were within normal ranges. Brain MRI showed multiple cerebral microbleeds and lacunar cerebral infarctions, while the size and density of the pituitary gland were normal (Figure 2). He received antiemetic drug treatment as well as intravenous fluids and sodium replacement. His body temperature returned to normal spontaneously, and serum sodium returned to normal level while the symptoms were relieved gradually.

Seventeen days later, it was planned to administer nivolumab to the patient in our hospital. He felt fatigue and anorexia once again the day before and increasing shortness of breath after admission to hospital. Physical examination showed fever, tachycardia, and dry rales of the lungs bilaterally. His blood pressure dropped to $52 / 26 \mathrm{mmHg}$ rapidly, arterial oxygen saturation $\left(\mathrm{SaO}_{2}\right)$ was $90 \%$, and he became somnolent gradually. Laboratory tests showed serum sodium was $115 \mathrm{mmol} / \mathrm{L}$; levels of plasma fibrinogen and D-dimer were elevated; CRP was $122.64 \mathrm{mg} / \mathrm{L}$; procalcitonin (PCT) was $0.703 \mathrm{ng} / \mathrm{mL}$ (normal range: $<0.5 \mathrm{ng} / \mathrm{mL}$ ); blood glucose level, blood leukocyte, and neutrophil counts were within normal ranges; and the ratio of eosinophils was 5.6\%. After oxygen inhalation and treatments of dopamine, antibiotic drug, low molecular weight heparin, sodium, and glucocorticoid, his symptoms of fever, dyspnea, and somnolence alleviated, with $\mathrm{SaO}_{2}>95 \%$. Levels of serum PCT and CRP decreased to normal. Physical examination showed dry rales disappearing
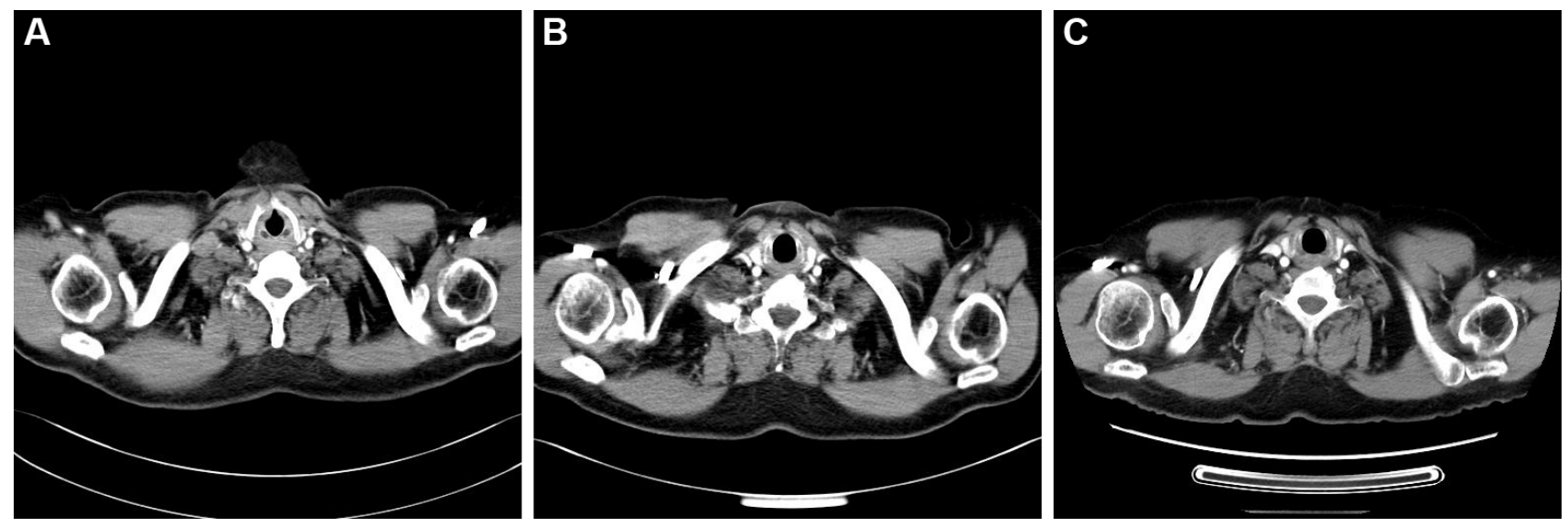

Figure I Computed tomography of the chest.

Notes: An enlarged right supraclavicular lymph node was observed before nivolumab treatment (A). The enlarged lymph node shrank significantly after two doses of nivolumab treatment (B). The shrunken lymph node remained stable for 6 months after discontinuation of nivolumab treatment (C). 

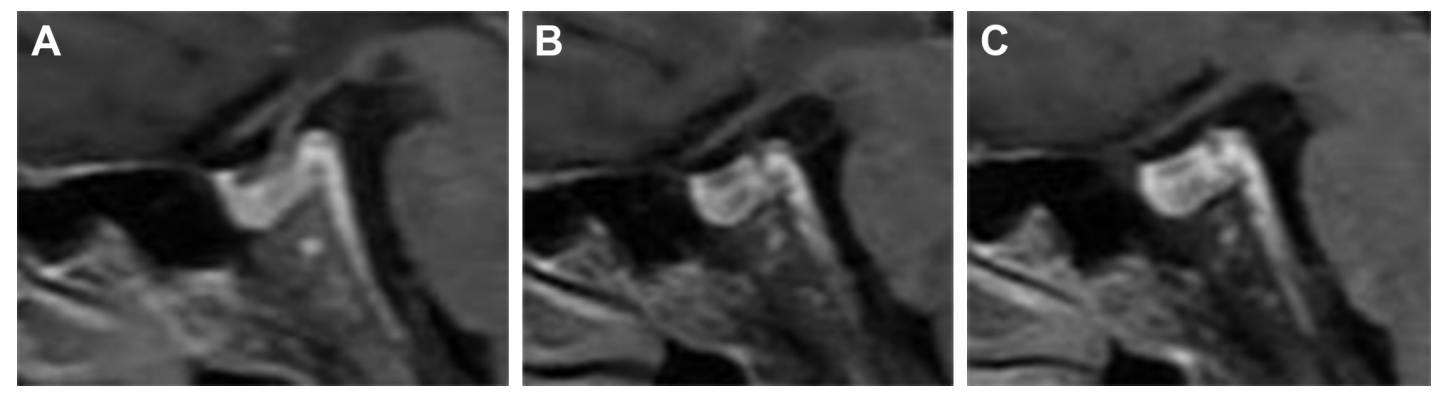

Figure 2 Brain MRI showed normal size and homogeneous enhancement of the pituitary gland.

Notes: Brain MRI before nivolumab treatment $(\mathbf{A})$, during the onset of hypophysitis (B), and 3 months after the diagnosis of hypophysitis $(\mathbf{C})$.

gradually, but his blood pressure and serum sodium level remained low. No signs of pulmonary artery embolism were detected by computed tomographic pulmonary angiography, and the mass size and atelectasis of lung were similar as before, while pleural and pericardial effusions increased. Laboratory tests of hormones revealed the followings: plasma ACTH at 8 am (normal range: $7.2-63.3 \mathrm{pg} / \mathrm{mL}$ ) was $2.43 \mathrm{pg} / \mathrm{mL}$; serum cortisol at 8 am (normal range: $4.4-19.9 \mu \mathrm{g} / \mathrm{dL}$ ) was $0.64 \mu \mathrm{g} / \mathrm{dL}$. Serum T3 (normal range: $0.92-2.79 \mathrm{nmol} / \mathrm{L}$ ) was $3.44 \mathrm{nmol} / \mathrm{L}$. Serum T4, FT4, FT3, TSH, growth hormone $(\mathrm{GH})$, and testosterone were within normal ranges. Luteinizing hormone (LH) (normal range: $1.24-8.62 \mathrm{mIU} / \mathrm{mL}$ ) was 17.98 $\mathrm{mIU} / \mathrm{mL}$, follicle-stimulating hormone $(\mathrm{FSH})$ (normal range: $1.27-19.26 \mathrm{mIU} / \mathrm{mL}$ ) was $52.46 \mathrm{mIU} / \mathrm{mL}$, and prolactin (normal range: $2.64-13.13 \mathrm{ng} / \mathrm{mL}$ ) was $24.96 \mathrm{ng} / \mathrm{mL}$. Hormone replacement with prednisone was given orally: $5 \mathrm{mg}$ at $8 \mathrm{am}$ and $2.5 \mathrm{mg}$ at $4 \mathrm{pm}$. After the treatment, his blood pressure and serum sodium increased to normal levels.

Despite the persistent low levels of serum ACTH and cortisol, the patient continued nivolumab therapy with longer intervals and received hormone replacement treatment as well. His clinical manifestations including fatigue, anorexia, and shortness of breath were alleviated. His blood pressure and serum sodium also were kept persistently normal. Seven months after the start of nivolumab treatment, he was diagnosed with primary hypothyroidism by low serum-free FT4 and elevated serum TSH. After taking oral levothyroxine, FT4 and TSH returned to normal levels. Nine months after the beginning of nivolumab treatment, he felt short of breath again, and the arterial blood gas showed hypoxemia with $\mathrm{SaO}_{2} 89 \%$. Immune-related pneumonitis was considered according to his medical history and the manifestation of chest CT. He was given intravenous methylprednisolone $1 \mathrm{mg} / \mathrm{kg}$ for 2 weeks. His dyspnea and hypoxemia were relieved afterward. Chest CT showed alleviated pneumonitis. Oral prednisone was then given and tapered gradually for the next 6-week period. Although nivolumab had not been used since the emergence of immune-related pneumonitis, his chest CT revealed that the tumor remained stable in the follow-up (Figure 1). Pituitary hormone levels were retested 10 months after the beginning of hormone replacement treatment and revealed as the following results: plasma ACTH and serum cortisol were below $1 \mathrm{pg} /$ $\mathrm{mL}$ and $0.91 \mu \mathrm{g} / \mathrm{dL}$ at $8 \mathrm{am}$, respectively. Serum T3, T4, FT3, FT4, TSH, testosterone, LH, FSH, GH, and prolactin were within normal ranges. The ratio of eosinophils was $2.8 \%$, and levels of serum CRP and sodium were normal.

\section{Discussion}

SCLC is an aggressive malignancy with rapid progression and poor prognosis. The prognosis is even worse in cases resistant to initial chemotherapy, and the mean survival time was only 5.4 months as shown in a systematic analysis. ${ }^{6}$ Recent clinical trials have shown that anti-PD-1 antibodies exhibited excellent efficacy in some resistant SCLC cases. ${ }^{7,8}$ Among 98 patients who received nivolumab treatment after progression upon standard chemotherapy, 10 had objective responses, another 22 patients got stable disease, and 6 patients had a duration of response longer than 6 months. ${ }^{7}$ Depending on this result, NCCN Guidelines had listed nivolumab as subsequent treatment for relapsed SCLC. Anti-PD-1 antibodies also showed excellent effects on other malignancies including advanced non-small-cell lung cancer (NSCLC), melanoma, renal-cell carcinoma (RCC), bladder cancer, head and neck cancer, and hepatocellular carcinoma. ${ }^{2}$ The disease of this patient progressed after receiving multiple chemotherapeutic agents and radiotherapy, which indicated poor response to ongoing subsequent chemotherapy and a dismal survival outlook. Up to now, the patient has been in a stable disease state for 15 months after nivolumab treatment and obtained survival benefit from the treatment.

Although exhibiting impressive efficacy, anti-PD-1 antibodies may manifest unique side effects related to autoimmune responses involving various organs including 
endocrine system. Adverse effects of endocrine system can be presented as hypophysitis, primary adrenal cortical dysfunction, thyroiditis, or type I diabetes mellitus. ${ }^{4,5}$

Autoimmune hypophysitis ( $\mathrm{AH})$ is a rare, immunemediated inflammatory disease, which is characterized by lymphocytic infiltration of the pituitary gland, resulting in deficits of one or multiple anterior pituitary hormones, which include ACTH, TSH, gonadotropins, prolactin, and GH. ${ }^{9}, 10$ Isolated ACTH deficiency (IAD) occurs when there is only a deficit in ACTH and is a potentially lethal condition with low cortisol production. ${ }^{9,11}$ Since ICPi (ipilimumab) was first used for melanoma treatment, this type of agent has become one of the causes of hypophysitis. The clinical characteristics of hypophysitis induced by anti-CTLA-4 antibodies are different from that induced by anti-PD-1 antibodies. Previous researches reported relatively high incidence $(8 \%-15 \%)$ of ipilimumab-induced hypopituitarism, ${ }^{12-14}$ while hypophysitis is rare among patients treated with nivolumab, with an incidence below $1 \% .{ }^{13,14}$ A recent analysis of 6,472 patients with different types of cancers showed that the incidence rate of hypophysitis was $3.2 \%$ and $0.4 \%$ for anti-CTLA-4 and antiPD-1 antibodies, respectively. ${ }^{15}$ The clinical presentations of ipilimumab-induced pituitary hormone disorder are similar to that of lymphocytic AH, with diffuse enlargement and abnormal enhancement of the pituitary gland on MRI, ${ }^{10,12,16,17}$ multiple anterior pituitary hormone deficits, and headache as the typical symptom. ${ }^{12,13,16,17}$ In contrast, normal size of the pituitary gland was described in most reported cases of nivolumab-induced hypophysitis (Table 1), ${ }^{18-20}$ just as shown in this case. However, considering ICPi-induced pituitary enlargement can be mild and transient, careful comparison between the MRI before and during the onset of hypophysitis is important. ${ }^{13,21}$ One combining analysis showed pituitary enlargement was resolved in all 40 patients with ipilimumabinduced hypopituitarism. ${ }^{13}$ Besides, MRI of the pituitary gland instead of the brain might be more precise in revealing mild change of the pituitary gland. Another characteristic of nivolumab-induced hypophysitis is that IAD is the most common manifestation of pituitary disorder, which is different from that of $\mathrm{AH}$ or ipilimumab-induced hypophysitis. In fact, IAD was demonstrated in most reported cases with nivolumab-induced hypophysitis (Table 1), ${ }^{10,18-22}$ just as in our case. The secretion of ACTH is the most frequent and initially affected pituitary hormone, ${ }^{9,10}$ which may explain the phenomenon of IAD. All the characteristics of nivolumabinduced hypophysitis, including IAD, normal pituitary size on MRI, and absence of headache, suggest that the inflammation of the pituitary gland may be milder in nivolumab-induced hypophysitis than that in ipilimumab-induced ones.

The pathological differences between hypophysitis induced by anti-CTLA-4 antibodies and anti-PD-1 antibodies are unclear. CTLA-4 antigen is found expressed on pituitary

Table I Characteristics of hormones and pituitary MRI in cases with ACTH deficiency

\begin{tabular}{|c|c|c|c|c|c|c|c|c|c|c|c|}
\hline \multirow[t]{2}{*}{ Cases } & \multirow[t]{2}{*}{$\begin{array}{l}\text { Agel } \\
\text { sex }\end{array}$} & \multirow[t]{2}{*}{$\begin{array}{l}\text { Cancer } \\
\text { type }\end{array}$} & \multirow[t]{2}{*}{$\begin{array}{l}\text { Abnormality of pituitary } \\
\text { gland on MRI }\end{array}$} & \multirow[t]{2}{*}{ АСТH } & \multirow[t]{2}{*}{ Cortisol } & \multicolumn{5}{|c|}{$\begin{array}{l}\text { Decrease of other anterior } \\
\text { pituitary hormones }\end{array}$} & \multirow[t]{2}{*}{ References } \\
\hline & & & & & & TSH & LH & FSH & GH & PRL & \\
\hline I & $61 / M$ & SCLC & No & $(+)$ & $(+)$ & $(-)$ & $(-)$ & $(-)$ & $(-)$ & $(-)$ & Present case \\
\hline 2 & $50 / M$ & Melanoma & $\begin{array}{l}\text { Mild enlargement of pituitary gland } \\
\text { and thickening of stalk }\end{array}$ & $(+)$ & $(+)$ & $(-)$ & $(-)$ & $(-)$ & $(-)$ & $(-)$ & 10 \\
\hline 3 & $39 / M$ & Melanoma & No & $(+)$ & $(+)$ & $(-)$ & $(-)$ & $(-)$ & $(-)$ & $(-)$ & 18 \\
\hline 4 & $50 / F$ & Melanoma & No & $(+)$ & $(+)$ & $(-)$ & $(-)$ & $(-)$ & $(-)$ & $(-)$ & 18 \\
\hline 5 & $74 / F$ & $\mathrm{RCC}$ & No & $(+)$ & $(+)$ & $(-)$ & $(-)$ & $(-)$ & $(-)$ & $(-)$ & 19 \\
\hline 6 & $55 / M$ & Melanoma & No & $(+)$ & $(+)$ & $(-)$ & $(-)$ & $(-)$ & $(-)$ & $(-)$ & 20 \\
\hline 7 & 68/M & Melanoma & No & $(+)$ & $(+)$ & $(-)$ & $(-)$ & $(-)$ & $(-)$ & $(-)$ & 20 \\
\hline 8 & $50 / M$ & Melanoma & No & $(+)$ & $(+)$ & $(+)$ & $(-)$ & $(-)$ & $(-)$ & $(-)$ & 20 \\
\hline 9 & $75 / M$ & NSCLC & No & $(+)$ & $(+)$ & $(-)$ & $(-)$ & $(-)$ & $(-)$ & $(-)$ & 20 \\
\hline 10 & $63 / F$ & NSCLC & $\begin{array}{l}\text { Slightly thickened hypophyseal } \\
\text { stalk }\end{array}$ & $(+)$ & $(+)$ & $(-)$ & $(-)$ & $(-)$ & $(-)$ & $(-)$ & 21 \\
\hline II & $54 / M$ & $\mathrm{RCC}$ & $\begin{array}{l}\text { Heterogeneous enhancement of } \\
\text { pituitary gland and enlargement } \\
\text { of basilar part }\end{array}$ & $(+)$ & $(+)$ & $(-)$ & $(-)$ & $(-)$ & $(-)$ & $(-)$ & 22 \\
\hline
\end{tabular}

Abbreviations: ACTH, adrenocorticotropic hormone; F, female; FSH, follicle-stimulating hormone; GH, growth hormone; LH, luteinizing hormone; M, male; NSCLC, nonsmall-cell lung cancer; PRL, prolactin; RCC, renal-cell carcinoma; SCLC, small-cell lung cancer; TSH, thyroid-stimulating hormone. 
endocrine cells, and high expression of CTLA-4 is associated with severe hypophysitis through type II and type IV hypersensitivities in cancer patients treated with antiCTLA-4 antibodies. ${ }^{14,23}$ On the contrary, the mechanism of nivolumab-induced hypophysitis remains unclear, and the expressions of PD-1 or its ligands on the pituitary gland are not evident. ${ }^{14}$ Recently, Fujimura et al described that serum levels of soluble CD163 (sCD163) and CXCL5 were strikingly upregulated in a case of melanoma with hypopituitarism induced by nivolumab. ${ }^{24}$ Expression of CD163 is restricted to monocyte/macrophage lineage, and levels of serum sCD163 increased in autoimmune diseases. CXCL5 is a biomarker of T-helper 17-mediated autoimmune diseases. ${ }^{24}$ These phenomena suggested the existence of autoimmune-like reactions in patients with hypophysitis induced by nivolumab.

Although definite diagnosis of hypophysitis is made by pathology through pituitary biopsy, clinical diagnosis can be made by comprehending the clinical manifestations and the pattern of pituitary hormone deficits. The present case manifested with anorexia, vomiting, fatigue, hypotension, hyponatremia, and low levels of cortisol, which are consistent with the manifestations of adrenal cortisol dysfunction. Somnolence and hypotension indicated the diagnosis of adrenocortical crisis, and the preexisting infection may be the potential trigger. Sustained low levels of plasma ACTH indicated secondary adrenocortical deficiency, probably due to nivolumab-induced hypophysitis. Another possible etiology is autoimmune disease. As the patient had no history of autoimmune disease and did not present with any related symptoms, autoimmune disease-related depression of the adrenocortical system was not likely, although relevant tests should be considered for better differential diagnosis in the future.

Another abnormality of our case was eosinophilia, which emerged 2 months before the onset of adrenocortical crisis and resolved 1.5 months after the hormone replacement. This phenomenon has been described in other reports, and some physicians suggest that eosinophilia may be an early predictor of adrenocortical insufficiency. ${ }^{25}$

An important issue is whether high-dose glucocorticoids should be given for the treatment of nivolumab-induced hypophysitis. Albarel et al observed that patients with ACTH deficiency induced by ipilimumab still had the deficiency in the end, irrespective of whether high dose or physiological replacement dose was given. ${ }^{17}$ Considering the potential adverse effects of high-dose hormones, we gave our patient a physiological replacement dose of prednisone, and his clinical disorders disappeared soon after prednisone administration. In fact, we noticed that most reported cases of nivolumab-induced hypophysitis were given replacement treatment. ${ }^{18,19,21,22,25}$ Another critical issue is whether nivolumab can continue to be used in patients experiencing serious hypophysitis. As adrenocorticotropic hormone deficiency induced by ICPi is usually permanent, ${ }^{17,26}$ nivolumab can be continued together with hormone replacement treatment. ${ }^{17,26}$ The patient in our report continued taking nivolumab treatment with longer intervals, and he did not feel uncomfortable, although his low ACTH and cortisol persisted. The European Society for Medical Oncology (ESMO) and American Society of Clinical Oncology (ASCO) have published clinical practice guidelines for the management of toxicities of immunotherapy in 2017 and 2018, respectively, ${ }^{4,5}$ and both suggest that serious toxicities warrant permanent discontinuation of the ICPi, with the exception of endocrinopathies that have been controlled by hormone replacement. The guideline also states that long-term hormone replacement therapy is required for most patients. ${ }^{4}$

As ICPis, including nivolumab, will be more and more widely used for the treatment of neoplasms, physicians should be familiar with the clinical presentation of hypophysitis induced by the agents and should consider the possibility of immune-related adverse events, such as hypophysitis, on the endocrine systems when patients feel uncomfortable or present with hyponatremia or hypotension. Appropriate and prompt treatment should be taken to prevent life-threatening adrenocortical crisis.

\section{Ethics approval}

We confirm that written informed consent was provided by the patient to have the case details and any accompanying images published. Institutional approval was not required to publish the case details.

\section{Disclosure}

The authors report no conflicts of interest in this work.

\section{References}

1. Dine J, Gordon R, Shames Y, Kasler MK, Barton-Burke M. Immune checkpoint inhibitors: an innovation in immunotherapy for the treatment and management of patients with cancer. Asia Pac J Oncol Nurs. 2017;4(2):127-135.

2. Gong J, Chehrazi-Raffle A, Reddi S, Salgia R. Development of PD-1 and PD-L1 inhibitors as a form of cancer immunotherapy: a comprehensive review of registration trials and future considerations. J Immunother Cancer. 2018;6(1):8.

3. Torino F, Corsello SM, Salvatori R. Endocrinological side-effects of immune checkpoint inhibitors. Curr Opin Oncol. 2016;28(4):278-287. 
4. Haanen J, Carbonnel F, Robert C, et al. Management of toxicities from immunotherapy: ESMO clinical practice guidelines for diagnosis, treatment and follow-up. Ann Oncol. 2017;28(suppl_4):iv119-iv142.

5. Brahmer JR, Lacchetti C, Schneider BJ, et al. Management of immunerelated adverse events in patients treated with immune checkpoint inhibitor therapy: American Society of Clinical Oncology Clinical Practice Guideline. J Clin Oncol. 2018;36(17):1714-1768.

6. Owonikoko TK, Behera M, Chen Z, et al. A systematic analysis of efficacy of second-line chemotherapy in sensitive and refractory smallcell lung cancer. J Thorac Oncol. 2012;7(5):866-872.

7. Ready N, Farago AF, de Braud F, et al. Third-line nivolumab monotherapy in recurrent SCLC: CheckMate-032. J Thorac Oncol. 2019;14(2):237-244.

8. Ott PA, Elez E, Hiret S, et al. Pembrolizumab in patients with extensivestage small-cell lung cancer: results from the phase Ib KEYNOTE-028 study. J Clin Oncol. 2017;35(34):3823-3829.

9. Caturegli P, Lupi I, Landek-Salgado M, Kimura H, Rose NR. Pituitary autoimmunity: 30 years later. Autoimmun Rev. 2008;7(8):631-637.

10. Okano Y, Satoh T, Horiguchi K, et al. Nivolumab-induced hypophysitis in a patient with advanced malignant melanoma. Endocr J. 2016;63(10):905-912.

11. Andrioli M, Pecori Giraldi F, Cavagnini F. Isolated corticotrophin deficiency. Pituitary. 2006;9(4):289-295.

12. Ryder M, Callahan M, Postow MA, Wolchok J, Fagin JA. Endocrinerelated adverse events following ipilimumab in patients with advanced melanoma: a comprehensive retrospective review from a single institution. Endocr Relat Cancer. 2014;21(2):371-381.

13. Faje A. Immunotherapy and hypophysitis: clinical presentation, treatment, and biologic insights. Pituitary. 2016;19(1):82-92.

14. Faje A. Hypophysitis: evaluation and management. Clin Diabetes Endocrinol. 2016;2(1):15.

15. Barroso-Sousa R, Barry WT, Garrido-Castro AC, et al. Incidence of endocrine dysfunction following the use of different immune checkpoint inhibitor regimens: a systematic review and meta-analysis. JAMA Oncol. 2018;4(2):173-182.
16. Carpenter KJ, Murtagh RD, Lilienfeld H, Weber J, Murtagh FR. Ipilimumab-induced hypophysitis: MR imaging findings. AJNR Am J Neuroradiol. 2009;30(9):1751-1753.

17. Albarel F, Gaudy C, Castinetti F, et al. Long-term follow-up of ipilimumab-induced hypophysitis, a common adverse event of the antiCTLA-4 antibody in melanoma. Eur J Endocrinol. 2015;172(2):195-204.

18. Kitajima K, Ashida K, Wada N, et al. Isolated ACTH deficiency probably induced by autoimmune-related mechanism evoked with nivolumab. Jpn J Clin Oncol. 2017;47(5):463-466.

19. Seki T, Yasuda A, Oki M, et al. Secondary adrenal insufficiency following nivolumab therapy in a patient with metastatic renal cell carcinoma. Tokai J Exp Clin Med. 2017;42(3):115-120.

20. Takaya K, Sonoda M, Fuchigami A, Hiyoshi T. Isolated adrenocorticotropic hormone deficiency caused by nivolumab in a patient with metastatic lung cancer. Intern Med. 2017;56(18):2463-2469.

21. Ohara N, Ohashi K, Fujisaki T, et al. Isolated adrenocorticotropin deficiency due to Nivolumab-induced hypophysitis in a patient with advanced lung adenocarcinoma: a case report and literature review. Intern Med. 2018;57(4):527-535.

22. Zeng MF, Chen LL, Ye HY, et al. Primary hypothyroidism and isolated ACTH deficiency induced by nivolumab therapy: case report and review. Medicine. 2017;96(44):e8426.

23. Caturegli P, Di Dalmazi G, Lombardi M, et al. Hypophysitis secondary to cytotoxic T-lymphocyte-associated protein 4 blockade: insights into pathogenesis from an autopsy series. Am J Pathol. 2016;186(12): 3225-3235.

24. Fujimura T, Kambayashi Y, Furudate S, et al. Isolated adrenocorticotropic hormone deficiency possibly caused by nivolumab in a metastatic melanoma patient. J Dermatol. 2017;44(3):e13-e14.

25. Ariyasu R, Horiike A, Yoshizawa T, et al. Adrenal insufficiency related to anti-programmed death-1 therapy. Anticancer Res. 2017;37(8): 4229-4232.

26. Torino F, Barnabei A, Paragliola RM, et al. Endocrine side-effects of anti-cancer drugs: $\mathrm{mAbs}$ and pituitary dysfunction: clinical evidence and pathogenic hypotheses. Eur J Endocrinol. 2013;169(6):R153-R164.
OncoTargets and Therapy

\section{Publish your work in this journal}

OncoTargets and Therapy is an international, peer-reviewed, open access journal focusing on the pathological basis of all cancers, potential targets for therapy and treatment protocols employed to improve the management of cancer patients. The journal also focuses on the impact of management programs and new therapeutic agents and protocols on

\section{Dovepress}

patient perspectives such as quality of life, adherence and satisfaction The manuscript management system is completely online and includes a very quick and fair peer-review system, which is all easy to use. Visit http://www.dovepress.com/testimonials.php to read real quotes from published authors. 